\title{
Tool and process condition monitoring
}

\section{Diagnostyka stanu narzędzi i procesu skrawania}

KRZYSZTOF JEMIELNIAK*

DOI: https://doi.org/10.17814/mechanik.2017.7.64

\begin{abstract}
Automatic tool condition monitoring is based on the measurements of physical phenomena which are correlated with this condition. There are numerous signal features (SFs) that can be extracted from the signal. As it is really not possible to predict which signal features will be useful in a particular case they should be automatically selected and combined into one tool condition estimation. This can be achieved by various artificial intelligence methods.
\end{abstract}

KEYWORDS: tool condition monitoring, signal processing

Striving for more and more reliable production processes creates the need for continuous monitoring. Automated tool and process diagnostics (DNiPS) is becoming an indispensable part of ensuring quality products and low cost. The diagnostic system should be able to detect catastrophic blunting of the blade (KSO) or excessive wear and tear of the tool, and to replace it with new ones in a timely manner, and to identify other abnormalities such as unwanted form of chips or selfexcited vibration. There are many extensive status reviews, such as [21], which present a variety of ideas and approaches to solve the problem of automated diagnostics. Still, the problem is still far from resolved because the existing systems are based on fuzzy, incomplete and randomly disturbed data coming from sensors that measure the phenomena occurring in the cutting zone.

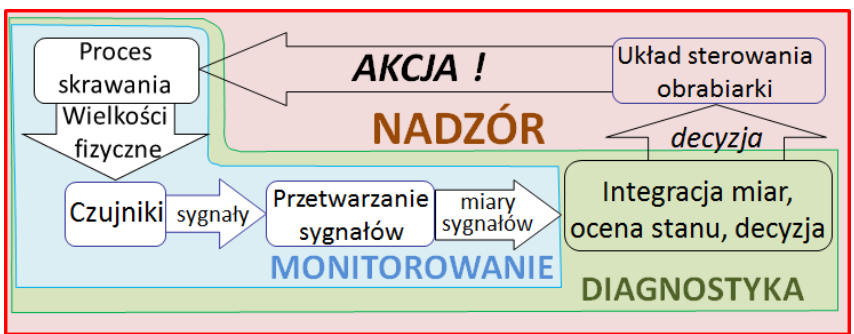

Fig. 1. Structure of monitoring, diagnostics and supervising system of tool and cutting process [6]

The purpose of this paper is to briefly present the issues of automatic monitoring, diagnostics, and tool

\footnotetext{
* Prof. dr hab. inż. Krzysztof Jemielniak (k.jemielniak@wip.pw.edu. pl) - Zakład Automatyzacji i Obróbki Skrawaniem, Wydział Inżynierii Produkcji Politechniki Warszawskiej
}

status monitoring and cutting. Concepts in this area are sometimes used interchangeably, and sometimes they are confused, so they are arranged in fig. 1 .

\section{Physical variables and sensors used in DNiPS}

The wear of the cutting tool results in the loss of blade material and in consequence, the change in its geometry, which in turn entails changes in cutting forces and derivative quantities (torque, engine power, deformation of machine parts or tool). Therefore, it is the cutting forces that are most commonly used in DNiPS. For turning, tapping and other machining - mainly using small pinch tools - force and cutting torque sensors are used. When milling, especially multi-axis, the force sensor installation is significantly impeded due to the complex movements of the tool and the object, therefore engine power sensors are relatively readily used [11].

Acoustic emission (AE) is the formation and propagation of decaying elastic waves, which is the result of the sudden release of energy of internal bonds damaged by deformation, cracking, etc. There are many $A E$ sources in the cutting zone such as: cracking of the workpiece, chip and tool; Tool friction with workpiece and chip with rake surface; Shear and plastic deformation of the workpiece $[10,12]$. Contrary to the misleading name, the $\mathrm{AE}$ frequencies used in DNiPS start at about $80 \mathrm{kHz}$ and reach $1 \mathrm{MHz}$, so they are very high - much higher than the audible band $(20 \mathrm{~Hz} \div 20 \mathrm{kHz})$, as well as the band of shear or vibration. Like the sound, the $A E$ spreads in solid bodies and liquids in accordance with all the laws of refraction and reflection, but air is the emission barrier. Emissions are very strong even with a small section of cut and almost imperceptible cutting forces. For this reason, AE is used for small overheads and wherever it is difficult to install a force sensor.

Mechanical vibrations are the least used variable [4], especially suitable for drilling small holes. As is well known, blunt drill drum, i.e. high frequency oscillation (in the order of several $\mathrm{kHz}$ ).

Sensors used in DNiPS are typically designed for these systems - adapted to the tough conditions of the cutting zone, and therefore resistant to coolant and striking shavings. The table lists the types of sensors offered by leading manufacturers of commercial DNiPS systems. 
TABLE. Sensors used in commercial DNiPS systems

\begin{tabular}{|c|c|c|c|c|c|c|c|}
\hline \multirow{2}{*}{\multicolumn{2}{|c|}{$\begin{array}{c}\text { Physical variable } \\
\text { (sensor type) }\end{array}$}} & \multicolumn{6}{|c|}{ Manufacturer of DNiPS systems } \\
\hline & & \multirow{2}{*}{ Artis } & \multirow{2}{*}{$\begin{array}{c}\begin{array}{c}\text { Bran- } \\
\text { kamp }\end{array} \\
\mathrm{O}\end{array}$} & \multirow{2}{*}{ Kistler } & \multirow{2}{*}{$\begin{array}{c}\text { Mon- } \\
\text { tronix }\end{array}$} & \multirow{2}{*}{\begin{tabular}{|c|c} 
Nord- \\
mann
\end{tabular}} & \multirow{2}{*}{\begin{tabular}{|c} 
Prome \\
-tec
\end{tabular}} \\
\hline . & Power & & & & & & \\
\hline & Torque & $\mathrm{O}$ & & $\mathrm{O}$ & $\mathrm{O}$ & & \\
\hline$\stackrel{\pi}{\geq}$ & Deformation & & $\mathrm{O}$ & $\mathrm{O}$ & $\mathrm{O}$ & & $\mathrm{O}$ \\
\hline$\frac{\Phi}{0} \frac{0}{0}$ & Distance & & & & & $\mathrm{O}$ & $\mathrm{O}$ \\
\hline$\stackrel{\bar{\sigma}}{c}$ & Force sensor & $\mathrm{O}$ & $\mathrm{O}$ & $\mathrm{O}$ & $\mathrm{O}$ & & $\mathrm{O}$ \\
\hline 离 & $\begin{array}{l}\text { Measuring pla- } \\
\text { te }\end{array}$ & & & 0 & O & & 0 \\
\hline \multirow[b]{3}{*}{ щ } & $\begin{array}{l}\text { Acoustic } \\
\text { emission }\end{array}$ & O & $\mathrm{O}$ & O & $\mathrm{O}$ & $\mathrm{O}$ & 0 \\
\hline & $\begin{array}{l}\text { Liquid AE } \\
\text { sensor }\end{array}$ & & & & & $\mathrm{O}$ & $\mathrm{O}$ \\
\hline & $\begin{array}{l}\text { Rotating AE } \\
\text { sensor }\end{array}$ & 0 & & & & $\mathrm{O}$ & $\mathrm{O}$ \\
\hline \multirow{3}{*}{ 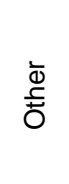 } & $\begin{array}{l}\text { Vibrations and } \\
\text { sound }\end{array}$ & & & 0 & 0 & & 0 \\
\hline & Camera & $\mathrm{O}$ & $\mathrm{O}$ & & & & \\
\hline & Laser & 0 & & & & 0 & \\
\hline
\end{tabular}

\section{Signal processing in DNiPS}

In the diagnostics of tool state and cutting process, a whole variety of signal processing techniques known from other fields, including general machine diagnostics, is used. Here are only some aspects of this treatment specific to DNiPS.

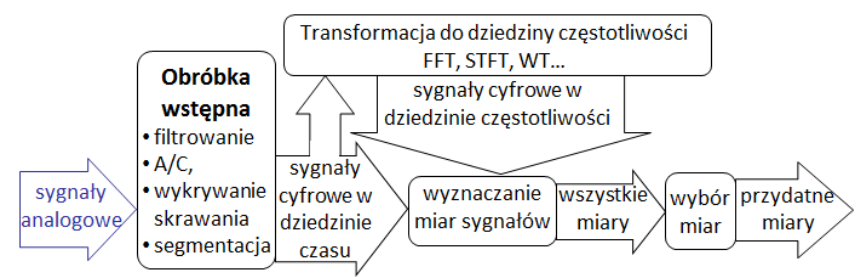

Fig. 2. General scheme of signal processing in DNiPS

- Initial signal preparation. Analog signals from the sensor are typically subjected to a pre-processing analogue, such as filtering to eliminate interference, or selecting an interesting frequency band or amplifier that matches the signal range to the $A / C$ converter. Analog pre-processed signals are converted to digital form and further processed to determine the characteristics of the signal that may serve as a symptom. However, automatic diagnostics can not be carried out on all (continuous) signals from sensors as they include signals collected during rapid movements and run and run. It is therefore necessary to automatically select fragments derived solely from cutting. The simplest and most common way of identifying cutting is to detect the excess by the operator of the limit value in the time window that he selects. This method is inconvenient, prone to interference and human error, and ineffective in the case of falling signals, which sometimes happens. It is more efficient to use low-pass filtered all available signals and their standard deviations, and to detect cuts based on the integration of these measures [3]. Moreover, online diagnostics based on long signal fragments is not possible for modern computers because of the need to analyze large numbers (even several hundred) of measurements. On the other hand, to analyze the degree of wear of the tool it is sufficient to analyze the short segments of the signals. Most signal segments are derived from set values. Measurement of the variation of the signal segment $B$ - i.e. the indicator of how much its effective value differs from neighboring fragments $A$ and $\mathrm{C}-$ may be the value of the factor $\mathrm{Fl}_{\mathrm{B}}[3]$ :

$$
F l_{B}=\left|\frac{\operatorname{RMS}[A]}{\operatorname{RMS}[B]}-1\right|+\left|\frac{\operatorname{RMS}[C]}{\operatorname{RMS}[B]}-1\right|
$$

All signal recorded during cutting is thus divided into seconds segments, from which the appropriate (small) number of segments least different from their neighbors are selected.

- Determination of time-domain signals. Preprepared, limited to cutting pieces of digital signal, timedomain measures are determined. Most commonly used are: mean, amplitude, range, peak factor, effective value, variance (or standard deviation), skewness, kurtosis, power, ratio or increment of signals. Some measures are typically used for vibration and $\mathrm{AE}$ signals - these are the number or rate of pulses or outbursts, and the pulse or burst width (percentage of time above the threshold) [10]. There are also time series modeling techniques: auto-regression (AR), moving average (MA), and combination of these techniques (ARMA) [15]. Signal measures are AR, MA or ARMA of the first or first and second order, less often the third, fourth and fifth order [20]. At present, the principal component analysis (PCA) and the singular spectrum analysis (SSA) are sometimes applied. PCA is one of the statistical methods of factor analysis, widely used to identify and reduce the size of systems. A data set consisting of $N$ observations, each of which includes $K$ variables, can be interpreted as a cloud of $N$ points in a $K$-dimensional space. PCA consists of such a rotation of the coordinate system that maximizes the variance of the first coordinate, then the variance of the second coordinate, etc. Converged co-ordinates are referred to as the loads of generated factors (main components).

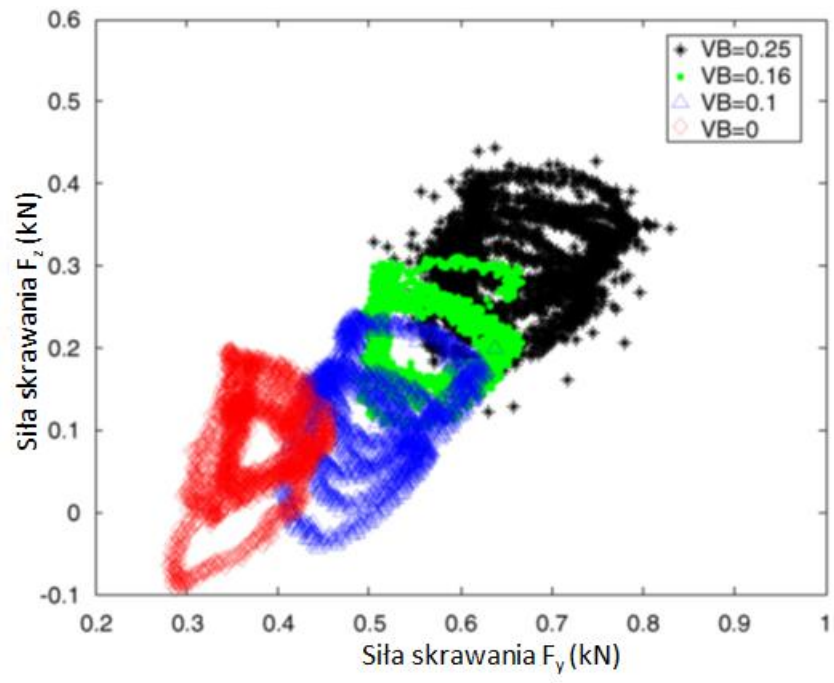

Fig. 3. Pie charts of cutting force signals at different blade wear times [17]

Shi and Gindy [18] used the signals of two perpendicular cutting force components for pulling into the blade wear diagnostics. The results of the 
measurements produced characteristic, distorted ellipses in the 2D space of shape and position clearly correlated with wear (fig. 3). The PCA designates the axial length $(a / b)$ and the ellipse $(\beta)$. The means of ellipses are the mean values of forces. Measurements of signals used for diagnostics are: $F_{y, \text { śr }}, F_{z, \text { śr }}, a, b, \beta$.

SSA is a technique of nonparametric time series analysis. It depends on the distribution of the signal into three independent components: the slow-moving trend representing the local mean, the difference between the signal and the average (oscillatory component) and the noise devoid of any structure [13]. These three components are treated as new signals, and they describe the basic measures discussed earlier (e.g. mean, variance, RMS, skewness, kurtosis).

Another interesting measure of the complexity of time series used in DNiPS is permutation entropy [11]. The smaller the permutation entropy, the more regular the time series. From the time series $x[i], i=1 \ldots n$ can be obtained $n$ ! permutation $\pi$ (settings in different order). The permutation entropy of such a series defines the relation:

$H_{p}(n)=\sum_{i=1}^{n !} p\left(\pi_{i}\right) \ln p\left(\pi_{i}\right)$

where: $p\left(\pi_{\mathrm{i}}\right)$ - relative permutation frequency of $\Pi_{\mathrm{i}}$ occurrence.

The normalized permutation entropy is then described by the formula:

$H_{p}=\frac{H_{p}(n)}{\ln (n !)}$

Li et al. [11] used this measure to detect KSO on the basis of the motor signal during finger milling. During normal cutting, the signal is regularly periodic and the $H_{\mathrm{p}}$ is low. After the occurrence of $\mathrm{KSO}$, this regularity falls significantly, which is accompanied by a jump in the $H_{\mathrm{p}}$ value.

- Determination of measure signals in the frequency or time and frequency domain. Digital signals in the time domain can be transformed into the frequency domain or time and frequency domain. Frequency signals are usually based on one of many available algorithms - the fast Fourier transform (FFT), which converts a time series of $N$ elements $x[n], n=0 \div$ $(N-1)$ to discrete representations in the domain Frequency $X[m], m=0 \div(N-1)$ :

$X[m]=\sum_{n=-\infty}^{N-1} x[n] e^{-j 2 \pi n m / N}$

The direct application of the Fourier coefficients $X[m]$ is impractical both for their considerable number and for the spectral leakage. The measure of this transformation is therefore measured: the amplitude of the dominant components, the power or energy in the selected bands, the average frequency, and the previously discussed measures that are used for time signals [4, 10, 20]. Although the signals coming from the cutting zone are generally non-stationary, the FFT averages the frequency components after the signal duration with a constant resolution equal to the inverse of that time. In order to include the temporal variability in time, a timefrequency analysis is used, such as the short-wave Fourier transform (STFT), in which the window $w[n]$ is moved along the time axis so that subsequent FFT transformations represent changes in frequency components in successive short periods of time. Marinescu and Axinte [12], who used $A E$ signals to detect $\mathrm{KSO}$ at milling, used the STFT to precisely determine the start and stop times of individual blades, i.e. signal segmentation.

STFT as a method of time-frequency analysis has a major drawback: the length of the window determines the resolution in both areas - time and frequency. Both can not be arbitrarily large and the improvement of one reflects on the deterioration of the other. The problem is opposite the wavelet transform (WT), where high frequencies are analyzed with narrow windows for better resolution in the time domain, and low - wide windows, giving better resolution in the frequency domain. Discrete wavelet transform (DWT) distributes the signal to scale factors (so-called approximations of $A$ ) and wavelengths (so-called $D$ details) using low and high pass filters. Filter responses are binary (every second sample is taken). The first approximation of $A_{1}$ is again decomposed into $A_{2}$ and $D_{2}$. In general, approximation $A_{j+1}$ and detail $D_{j+1}$ can be described as:

$A_{j+1}[n]=\sum_{k=-\infty}^{\infty}\left(A_{j}[k] h[2 n-k]\right)$

$D_{j+1}[n]=\sum_{k=-\infty}^{\infty}\left(A_{j}[k] g[2 n-k]\right)$

where $h$ and $g$ are pulse responses of low and high pass filters (scaling and wavelet functions).

Another type of WT is packet wavelet transform (WPT), in which further decomposition is subject not only to approximation but also to detail. This allows for more useful signal measurements. As in the case of Fourier transforms, wavelet coefficients are generally not used directly, but they determine the measures used for timedomain signals.

Another relatively new time-frequency analysis method used in DNiPS is the Hilbert-Huang transform (HHT), which is particularly useful for non-stationary and nonlinear signals that can change even within a single oscillation period. Unlike STFT or WT, the HHT method is rather an algorithm (due to the empirical approach) than a theoretical one. It uses two signal processing techniques (two steps):

- empirical decomposition of the signal into nonlinear modal components (as opposed to typical harmonic components, these functions may vary in amplitude and frequency in the time domain),

- a Hilbert transform that determines changes in amplitude and frequency of signal components over time and produces the resulting time-frequency distribution (Hilbert's spectrum).

Peng [13] applied this method for detecting KSO based on the cutting force signals in milling. KSO can be detected directly in the Hilbert spectrum or through modal components associated with characteristic milling frequencies. After the KSO occurs, the energy of the characteristic components changes in the opposite direction, unlike changing the cutting parameters.

- Selection of signal measures. As it is generally not possible to reliably monitor the state of the tool and the cutting process on the basis of a single signal measure, and it is not possible to predict in advance what signal measures will be useful in a particular application, it is commonly believed that the determination of a sufficiently large number of measures is a key a problem in any DNiPS system. The number of 
selected signal measures must be large enough to compensate for accidental interferences (which are unavoidable) and, on the other hand, to remove unnecessary or uncorrelated measures. While the measurement of signals is a problem well known for many other uses, the choice of measure is often underestimated, although its importance for the efficiency of the diagnostic system is enormous. In industrial applications, the choice of measures must be carried out automatically, with or without operator input. Meanwhile, according to a very interesting classification of the procedures for the selection of measures for the diagnosis of blade wear at turning, presented by Sick [19], $38 \%$ of the 138 analyzed publications of signal measurements were selected without any justification (or based on bibliographic data), 26\% They were defined after the analysis of the measured signals, and in $21 \%$ the most useful measures were selected without taking into account the resulting wear assessment of the blade. Only in $15 \%$ of analyzed publications, the optimal set of measures was determined after analyzing the impact of this choice on the wear assessment of the blade.

In laboratory diagnostic systems, as a measure of blade wear, geometric measures of wear are generally used (the width of the $V B_{B}$ application surface or the depth of the $K T$ crater). However, in industrial conditions such indicators are rarely used. For this reason, it was proposed in [7] that a part of its durability $(\Delta T)$ was used as a measure of blade wear, defined as the ratio of the previous cutting time $(t)$ to the total shelf life $(T): \Delta T=t / T$. This makes it possible to evaluate the state of the tool from the wear indicator, to use several indicators at the same time or from discrete indicators, blunting, burrs, chipping, blade breaking, etc. [9].

Evaluation of the usefulness of signal measures (MS) depends on their further use and can be carried out in many ways. Monitoring itself is always based on some model of the measure of the state of the tool state (fig. 4). The model may be e.g. approximation of a second or third degree polynomial or a low pass filtered waveform. The diagnostic system must determine this dependency (model parameters) and then invert it to determine the state of the tool (fig. $4 b$ ).

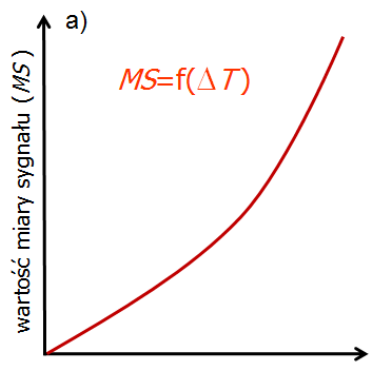

stan narzędzia $(\Delta T)$

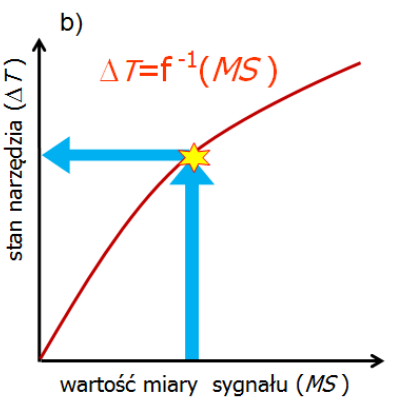

Fig. 4. Model of dependence of signal measure on the state of tool (a) and its use in diagnostics of this state $(b)$

Some researchers (e.g. $[15,16])$ to find the best measure of blade wear, used the Pearson $r$ correlation coefficient between the measure and the wear value assuming the lower the value, the less chance that the measure would show any dependency on the blade wear. It is ignored that even if the measure is perfectly correlated with wear, but the correlation is nonlinear, the correlation coefficient $r<1$.

In order to avoid the uncertainty associated with the modeling of the relation of the measure of the state of the tool in [9], the use of the determinant $R_{\mathrm{s}}{ }^{2}$ was proposed. It indicates the extent to which any model yields experimental values, or - in other words - how much this model of dependence of an MS signal on a tool state better describes the actual course than its mean value $\overline{M S}$. The determination coefficient is expressed by the relation:

$R_{S}^{2}=\frac{C S K-R S K}{C S K}=\frac{\sum_{i}\left(M S_{i}-\overline{M S}\right)^{2}-\sum_{i}\left(M S_{i}-M S_{m i}\right)^{2}}{\sum_{i}\left(M S_{i}-\overline{M S}\right)^{2}}$

where: $C S K=\sum_{i}\left(M S_{i}-\overline{M S}\right)^{2}-$ total sum of squares; $R S K=\sum_{i}\left(M S_{i}-M S_{m i}\right)^{2}-$ residual sum of squares; $C S K-$ $R S K=Z N K-$ abolished the sum of the squares; $M S_{i}, M S_{m i}$ - successive values of measured and modeled values in the normalized points of the used part of the shelf life $(i=$ $0 \div 100) ; \overline{M S}$ - average measure.

Measures, for which the $R_{\mathrm{s}}{ }^{2}$ value is higher than the assumed threshold, e.g. $R_{\mathrm{s}}^{2}>0.4$, are accepted.

The tool status monitoring system should be ready for use at the end of the first shelf life. However, after the second shelf life has expired, the selection can be repeated using all recorded data. $R_{\mathrm{s}}{ }^{2}$ coefficients are then calculated for two periods and averaged. It is also possible to introduce a second, even more important, criterion for the usefulness of measures - repetition. It is determined using the second determinant $R_{r}^{2}$, which compares the models created automatically on the basis of the first and second shelf life:

$R_{r}^{2}=\frac{\sum_{j} \sum_{i}\left(M S_{m j i}-\overline{M S}_{2 m}\right)^{2}-\sum_{j} \sum_{i}\left(M S_{m j i}-\overline{M S}_{m i}\right)^{2}}{\sum_{j} \sum_{i}\left(M S_{m j i}-\overline{M S} \bar{S}_{2 m}\right)^{2}}$

where: $M S_{m j i}-$ modeled value of the $M S_{m}$ measure at the $i$-th point $(i=0 \div 100)$ and the $j$-th term of life $(j=$ $1 \div 2), \quad \overline{M S}_{m i}=\frac{1}{2} \sum_{j} M S_{m j i}-$ average value of the $M S_{m}$ model at the $i$-th point, $\overline{M S}_{2 m}=$ $\frac{1}{202} \sum_{j} \sum_{i} M S_{m j i}$ - average of all $M S_{m}$ values for the two shelf life periods.

Measures, for which $R_{\mathrm{r}}^{2}$ is higher than the assumed threshold (e.g. $R_{r}^{2}>0.6$ ) are considered sufficiently reproducible. Of course, the procedure can be repeated after the third shelf life.

Al-Habaibeh and Gindy [1] searched for signal values depending on the milling condition, using the mean values of the dependencies obtained from the orthogonal Taguchi matrices as indicators of the usefulness of a combination of measurements from a particular sensor, to detect dullness of the tool (the stronger dependence, the more useful the sensor). Sun et al. [20] identified the most useful measures using Bayesian model and support vector machine (SVM). An error was evaluated for the condition of the tool modeled using the selected measure with the actual state (acute), and the worst measures were eliminated.

Another way to verify the usefulness of measures is statistical overlap factor (SOF), which determines the degree of separation of measure values corresponding to sharp and blunt tools [17]. This coefficient is defined as:

$S O F=\left|\frac{\overline{M S}_{1}-\overline{M S}_{2}}{\left(\sigma_{1}-\sigma_{2}\right) / 2}\right|$

where: $\overline{M S}_{1}, \sigma_{1}, \overline{M S}_{2}, \sigma_{2}$ - the mean and standard deviation of the measurement values for sharp (1) and blunt tool (2), respectively. 
The authors [17] noted that in the automatic selection, measures are often too close or similar to one another, which contradicts the goal of integrating different measures. In such cases, they recommended "engineering evaluation", which limits the automatism of choice and forces manual intervention by the operator or rather the researcher. Such a procedure is not acceptable in industrial conditions, so in [9] the measures that met the condition of bonding to the blade state $\left(R_{\mathrm{s}}^{2}>0.4\right)$ were sorted in decreasing respect to $R_{\mathrm{s}}{ }^{2}$, then the first (best) and the Pearson correlation coefficient $r^{2}$ between this measure and each next. Measures for which $r^{2}>0.8$ were rejected as too closely related to the best. Of the remaining measures again, the best was selected and the measures associated with it were rejected.

Binsaeid et al. [2] also assessed the suitability of the measure based on the correlation of a single measure with the state of the blade and correlation between the measures. The coefficient of usefulness of the measure was determined by the relation:

$m=\frac{N \bar{r}_{c f}}{\sqrt{N+N(N-1) \bar{r}_{f f}}}$

where: $N$ - number of measures, $\bar{r}_{c f}, \bar{r}_{f f}-$ mean correlation of tool-measure and measure-measure.

Correlation was investigated by entropy measures. The high score is achieved by measures well correlated with the state of the tool and at the same time poorly correlated among each other.

\section{Integration of measures, state assessment}

Measures of signals useful for tool status determination, automatically determined according to the algorithms described above, must be integrated, which means that the tool state must be determined based on the part of the blade life span used. There are many methods of such integration, such as statistical modeling, autoregressive methods, pattern recognition methods, or expert systems. Especially artificial intelligence methods such as neural networks, genetic algorithms or fuzzy logic, as well as hybrids of these methods are particularly popular [5, 21]. The most popular are neural networks, and among them multilayer perceptron with the method of backward error propagation [22]. As a rule, a single neural network is used to input selected signal measurements, and an estimate of the selected blade consumption index is obtained. In [8], it has been shown that the number of learning data obtained during one blade life span is too small relative to the required network size required to map the complex dependencies of the plurality of measures on the tool state. Similar to other methods of artificial intelligence - large numbers of measurements require a large amount of learning data available only after a few or even a dozen or so periods of durability. This problem can be solved using hierarchical algorithms [8,9], where the state of the tool is evaluated first based on each selected measure or several measures separately, and then integrates the obtained scores, e.g. by averaging.

\section{Example of a tool status diagnostic system operation}

In order to illustrate the issues discussed in this paper, we present work of the tool status diagnostic system on the example of ADONiS system (acronym for automatic diagnosis of cutting tool blades), developed by the team of the Automation and Machining Department of the Faculty of Production Engineering, Warsaw University of Technology [3, 8, 9]. The study was conducted on a Venus 450 lathe with a cutting force sensor (Kistler 9601A31) installed under the transverse slide (fig. 5a) and an acoustic emission sensor (Kistler 7815B121) located on the support. Four signals were measured: $F_{c}$, $F_{\mathrm{f}}$ and $F_{\mathrm{p}}$, as well as $A E_{\mathrm{RMs}}$. Workpieces were steel rollers with a diameter of $\varnothing 160 \mathrm{~mm}$. The operation plan (fig. $5 b$ ) consisted of 22 consecutive coarse passes with $a_{\mathrm{p}}=1.5 \mathrm{~mm}$ (13 passes) and $a_{\mathrm{p}}=2 \mathrm{~mm}$ (9 passes), $f=$ $0.1 \mathrm{~mm} / \mathrm{rev}$ and $v_{\mathrm{c}}=150 \mathrm{~m} / \mathrm{min}$ - and one finishing pass with the same feed rate and cutting speed, but with variable cutting depth. SCGCL turning knives with CNMG 10408 BP30A cemented carbide blades were used. Machining of one item lasted $4.6 \mathrm{~min}$, including a cutting time of $3.6 \mathrm{~min}$. Eight tools were dugged after processing $8,10,10,12,10,9,14$, and 10 items respectively.

Cutting detection was based on the low-pass filtered values of all four signals. After the start of the working feed, and prior to cutting, the system calculated the mean values of the signals and subtracted them by removing the offset.

The standard deviation values at tool run time $\sigma_{0}$ are used to automatically determine the thresholds of filtered $S_{f}$ values and their standard deviations $\sigma_{c}$. Exceeding any signal of the appropriate threshold $\left(S_{f}>5 \sigma_{0}\right.$ or $\left.\sigma_{\mathrm{c}}>3 \sigma_{0}\right)$ means initiating the cutting. The cutting break is detected when all measures (mean values and standard deviations) that exceed their thresholds fall below the thresholds. All of these activities are performed automatically, with or without operator knowledge.

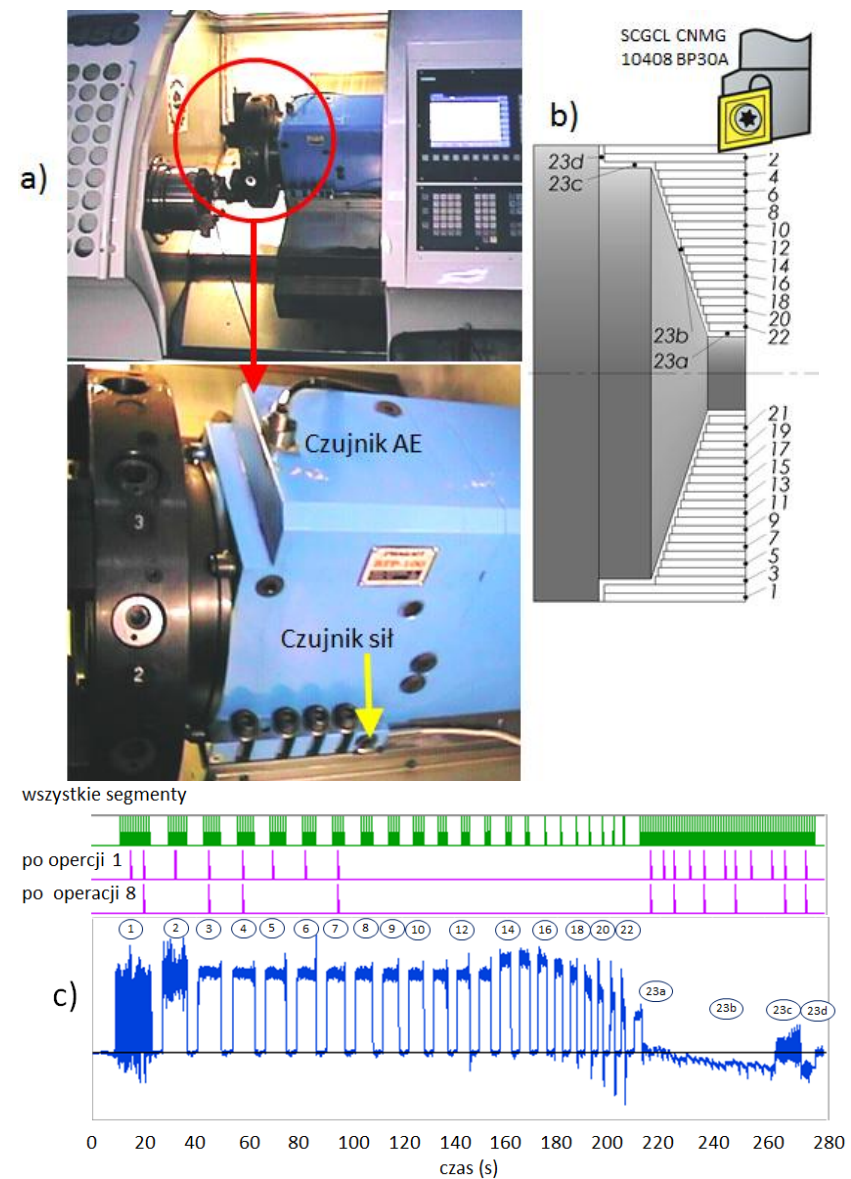

Fig. 5. Method of installing the sensors (a), operation plan (b) and elimination of segments $(c)$ 
Fig. $5 c$ shows an exemplary waveform of the $F_{c}$ force and the results of the segment selection procedure. After the first operation (top row in fig. 5c), 165 one-second segments were identified during the cutting, 19 of which were selected, taking into account the criterion of invariance with respect to the neighbors - equation (1). After seven operations, there were already $19 \times 7=133$ segments in the memory of the system, so more than the maximum of 128 , so each of the two selected better (more close to the neighbors), leaving 10 segments per operation.

Each of the four measured signals was automatically processed using packet wavelet transform for 14 coefficients (approximations and details - Equations (5) and (6)), which automatically calculated the following measures:

- logarithmic energy (e.g. $F_{\mathrm{c} / \mathrm{DD} . \mathrm{E}}-$ for coefficient $D D$ of signal $F_{\mathrm{c}}$ ),

- effective value (e.g. $F_{\text {f/ADA.RMS }}$ - for coefficient $A D A$ of signal $F_{\mathrm{f}}$ ),

- standard deviation (e.g. $F_{\mathrm{f} / \text { A.st dev }}$ for coefficient $A$ of signal $F_{\mathrm{f}}$ ),

- mode (e.g. $A_{\mathrm{E} / \mathrm{s} \text {.mode }}$ for the original $A E$ signal),

- explosion rates 1,2 and 3 - number of thresholds exceeded $30 \%, 50 \%$ and $70 \%$ of maximum value (e.g. $\left.F_{\mathrm{p}, \mathrm{ADA} \text {,Count1 } 1}\right)$,

- width of outbreaks 1,2 and 3 - percentage of time above thresholds (e.g. $F_{\text {D.ADA.Pulse } 1}$ ).
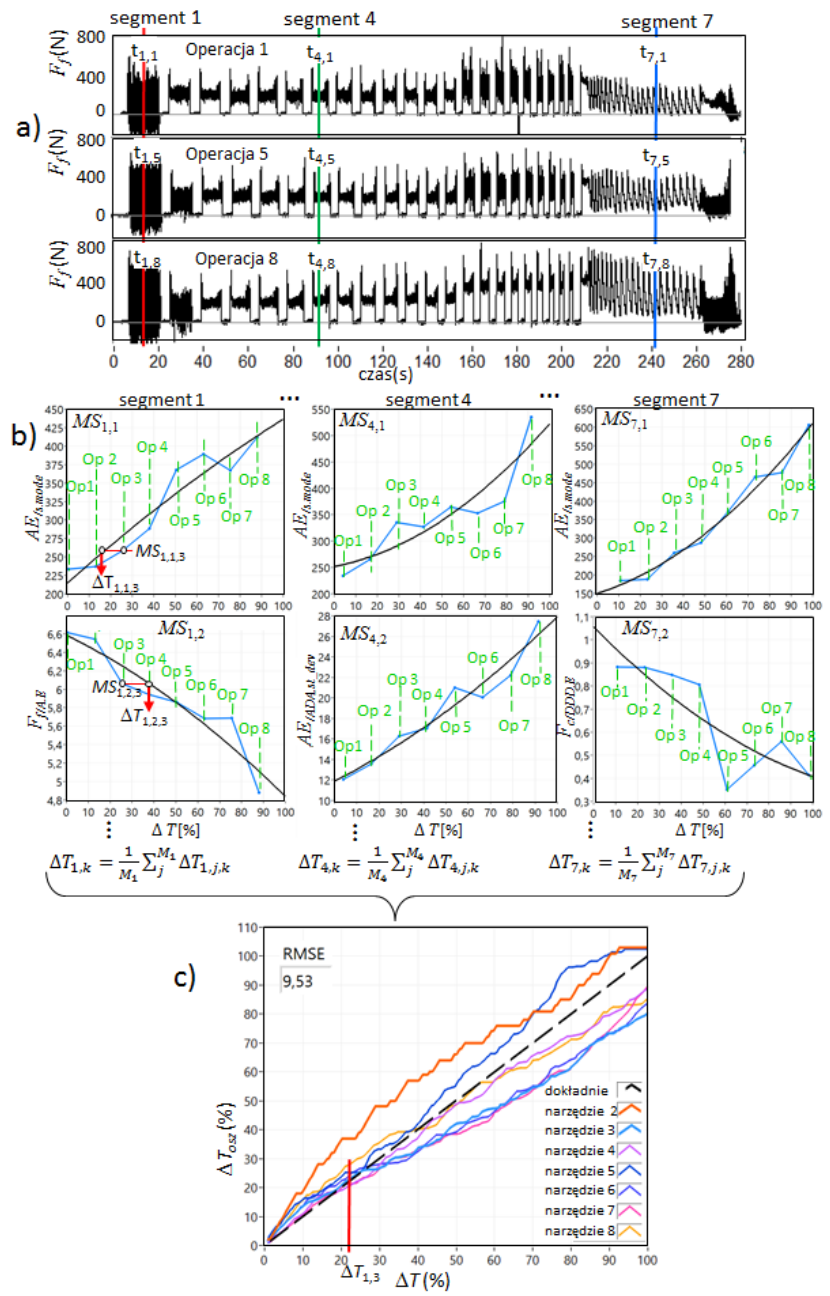

Fig. 6. Signals of the feed force in three operations (a); examples of signal measures selected and modeled by ADONiS in sections 1,4 and $7(b)$; estimation of the used part of blade life span in periods $2 \div 8(c)$

Since all procedures are automatic (no operator involvement required), a different number of other measures are assigned to each segment. Their examples in three of the eight operations of the first shelf life are shown in fig. $6 b$ (blue lines). At the end of this period for each segment, ADONiS set a separate model based on the approximation of the second degree polynomial (black continuous lines in fig. 6b). During operation of the next tools, the system worked in a diagnostic mode. After collecting data from each selected segment, the system determined the part of the shelf life used separately from each measure.

For example, for the first segment in the third operation, $\Delta T$ estimated from the first and second measures $-A \mathrm{E}_{/ \mathrm{s} \text {.mode }}$ and $F_{f / A . E}-$ were respectively $\Delta T_{1,1,3}$ and $\Delta T_{1,2,3}$ (fig. $6 b$ ). After their averaging, the estimated portion of the shelf life of the blade at that moment (for the segment) was estimated:

$\Delta T_{i, k}=\frac{1}{M_{i}} \sum_{j}^{M_{i}} \Delta T_{i, j, k}$

where: $i$ - segment number, $j$ - number of measure for the segment, $k$ - number of operations, $M_{i}-$ number of measures in the segment $i$.

Results of the supervising are shown in fig. $6 c$ as used parts of the shelf life estimated by ADONiS $-\Delta T_{\text {osz }}$ as a function of experimental values $\Delta T$ for tools $2 \div 8$ (the first shelf life was used to teach the system).

The accuracy of the used part of the shelf-life was assessed by means of a mean square error ( $R M S E)$ :

$R M S E=\sqrt{\frac{1}{n} \sum\left(\Delta T_{o s Z}-\Delta T\right)^{2}}$

Because $\Delta T$ is expressed as a percentage, RMSE can be interpreted as an average percentage error estimate. Its value is also given in fig. $6 c$.

Presented estimates of the tool state during operation is especially important in the aviation industry, where machining of one item may take several minutes, and a number of tools (e.g. [9]) need to be performed for one operation.

\section{Conclusions}

The paper discusses issues related to the tool status diagnostics, in general. It is particularly important to designate as many measures as possible from all the available signals and their automatic (without operator involvement) evaluation and selection. Measures integration can easily be done in a variety of ways, but it is a good idea to use a hierarchical algorithm, where the first step evaluates the state of the tool on the basis of single or few measures and these measures are integrated in the second one.

\section{REFERENCES}

1. Al-Habaibeh A., Gindy N. "A new approach for systematic design of condition monitoring systems for milling processes". Journal of Materials Processing Technology. 107 (2000): pp. 243-251.

2. Binsaeid S., Asfoura S., Chob S., Onarc A. "Machine ensemble approach for simultaneous detection of transient and gradual anomalies in milling using multisensor fusion". Journal of Materials Processing Technology. 209 (2009): pp. 4728-4738.

3. Błażejak K., Bombiński S., Nejman M., Jemielniak K. "Sensor signal segmentation for tool condition monitoring". Procedia CIRP. 46 (2016): pp.155-160.

4. El-Wardany T.I., Gao D., Elbestawi M.A. "Tool condition monitoring in drilling using vibration signature analysis". International Journal of Machine Tools and Manufacture. 36 (1996): pp. 687-711.

5. Halgamuge S.K., Glesner M. "NNs in designing fuzzy systems for real world applications". Fuzzy Sets and Systems. 65 (1984): pp. 112. 
6. Jemielniak K. „Automatyczna diagnostyka stanu narzędzia $i$ procesu skrawania”. Warszawa: Oficyna Wydawnicza Politechniki Warszawskiej, 2002.

7. Jemielniak K. "Tool wear monitoring based on a non-monotonic signal feature". J. Engineering Manufacture. Part B. 220, 2 (2006): pp. 163-170.

8. Jemielniak K., Bombiński S. "Hierarchical strategies in tool wear monitoring". Proc. IMechE. 220/B (2006): pp. 375-381.

9. Jemielniak K., Kossakowska J., Urbański T., Bombiński S. "Tool condition monitoring based on numerous signal features". International Journal of Advanced Manufacturing Technology. 59 (2012): pp. 73-81.

10. Li X. "A brief review-acoustic emission method for tool wear monitoring in turning". International Journal of Machine Tools and Manufacture. 42 (2002): pp. 157-165.

11. Li X., Ouyang G., Liang Z. "Complexity measure of motor current signals for tool flute breakage detection in end milling". International Journal of Machine Tools and Manufacture. 48 (2008): pp. 371-379.

12. Marinescu I., Axinte D. "A time-frequency AE-based monitoring technique to identify workpiece surface malfunctions in milling with multiple teeth cutting simultaneously. International Journal of Machine Tools and Manufacture. 49 (2009): pp. 53-65.

13. Peng Y. "Empirical model decomposition based time-frequency analysis for the effective detection of tool breakage". Journal of Manufacturing Science and Engineering. 128, 1 (2006): pp. 154166.

14. Salgado D., Alonso F. "Tool wear detection in turning operations using singular spectrum analysis". Journal of Materials Processing Technology. 171 (2006): pp. 451-458.

15. Quan Y., Zhoub M., Luo Z. "On-line robust identification of tool wear via multi-sensor NN fusion". Engineering Applications of Artificial Intelligence. 11 (1998): pp. 717-722.

16. Scheffer C., Heyns P.C. Wear monitoring in turning operations using vibration and strain measurements". Mechanical Systems and Signal Processing. 15, 6 (2001): pp. 1185-1202.

17. Scheffer C., Heyns P.C. "An industrial tool wear monitoring system for interrupted turning". Mechanical Systems and Signal Processing. 18 (2004): pp. 1219-1242.

18. Shi D., Gindy N.N. "Tool wear predictive model based on least squares support vector machines". Mechanical Systems and Signal Processing. 21 (2007): pp. 1799-1814.

19. Sick B. "On-line and indirect tool wear monitoring in turning with artificial neural networks: a review of more than a decade of research". Mechanical Systems and Signal Processing. 16, 4 (2002): pp. 487-546.

20. Sun J., Hong G.S., Rahman M., Wong Y.S. "Identification of feature set for effective tool condition monitoring by AE sensing". International Journal of Production Research. 42, 5 (2004): pp. 901-918.

21. Teti R., Jemielniak K., O'Donnell G., Dornfeld D. "Advanced monitoring of machining operations (keynote paper)". CIRP Annals - Manufacturing Technology. 59, 2 (2010): pp. 717-739.

22. Teti R., Kumara S.R.T. "Intelligent computing methods for manufacturing systems". STC-O KN Paper. CIRP Annals. 46/2 (1997): pp. 629-652. 